Portland State University

PDXScholar

Electrical and Computer Engineering Faculty

Publications and Presentations

Electrical and Computer Engineering

6-2014

\title{
Coherence Extrapolation for Underwater Ambient
}

Noise

Jorge Quijano

Portland State University

Stan E. Dosso

University of Victoria

Martin Siderius

Portland State University, siderius@pdx.edu

Lanfranco Muzi

Portland State University

Follow this and additional works at: https://pdxscholar.library.pdx.edu/ece_fac

Part of the Electrical and Computer Engineering Commons

Let us know how access to this document benefits you.

Citation Details

Quijano, J. E., Dosso, S. E., Siderius, M., \& Muzi, L. (2014). Coherence extrapolation for underwater ambient noise. Journal Of The Acoustical Society Of America, 135(6), EL318-EL323.

This Article is brought to you for free and open access. It has been accepted for inclusion in Electrical and Computer Engineering Faculty Publications and Presentations by an authorized administrator of PDXScholar. Please contact us if we can make this document more accessible: pdxscholar@pdx.edu. 


\title{
Coherence extrapolation for underwater ambient noise
}

\author{
Jorge E. Quijano a) and Stan E. Dosso \\ School of Earth and Ocean Sciences, University of Victoria, 3800 Finnerty Road, Victoria, \\ British Columbia V8P 5C2, Canada \\ jorgeq@uvic.ca,sdosso@uvic.ca \\ Martin Siderius and Lanfranco Muzi \\ Northwest Electromagnetics and Acoustics Research Laboratory, Portland State University, \\ 18 SW 4th Avenue, Portland, Oregon 97201 \\ siderius@pdx.edu,lan.muzi@gmail.com
}

\begin{abstract}
This paper considers extrapolation of the vertical coherence of surface-generated oceanic ambient noise to simulate measurements made on a longer sensor array. The extrapolation method consists of projecting the noise coherence measured with a limited aperture array into the domain spanned by prolate spheroidal wave functions, which are an orthogonal basis defined by array parameters and the noise frequency. Using simulated data corresponding to selected multi-layered seabeds as ground truth, the performance of the extrapolation method is explored. Application of the technique is also demonstrated on experimental data.
\end{abstract}

(C) 2014 Acoustical Society of America

PACS numbers: $43.60 . \mathrm{Uv}, 43.60 . \mathrm{Rw}, 43.30 . \mathrm{Nb}$ [DC]

Date Received: February 14, 2014 Date Accepted: April 24, 2014

\section{Introduction}

In shallow water, the ambient noise field resulting from wind-driven breaking waves and rain interacts with the bottom and hence can be inverted for remote exploration of seabed sediment properties. ${ }^{1-8}$ The noise field is normally measured at a vertical line array (VLA), and the resolution of geoacoustic parameters inferred from such data is strongly affected by the array aperture. This paper describes a technique to extend the aperture of a VLA by extrapolating the noise coherence measured at an $N$-element VLA to approximate the coherence of an effective $N_{e}$-element VLA where $N_{e}>N$ with the same element spacing.

For inference of seabed parameters from surface-generated ambient noise, two lines of processing focusing on different features of the seabed have been developed: The coherent approach ${ }^{5}$ (referred to as the passive fathometer) that uses the amplitude and phase information of surface-generated and seabed-reflected noise wavelets to produce an image of the seabed layering structure and the incoherent approach ${ }^{3,4}$ that takes the ratio of the power of upward-to-downward noise wavelets to approximate the seabed power reflection coefficient. Both processing approaches apply beamforming to determine angular distribution of the noise.

Despite the advantage of larger arrays in terms of beamforming resolution, short arrays offer significant practical advantages in field measurements such as easy deployment, robustness to array deformation due to currents, as well as being amenable for integration into autonomous vehicles. For this reason, methods for synthetic extension of the array aperture are attractive. While a heuristic method based on zeropadding of the noise coherence has been suggested ${ }^{9}$ to enhance features of the

\footnotetext{
${ }^{\text {a) }}$ Author to whom correspondence should be addressed.
} 
estimated power reflection coefficient, the goal of this paper is to describe a formal mathematical approach for noise-covariance extrapolation.

\section{Surface-generated ambient-noise coherence}

It has been shown that under mild conditions regarding the separation of the VLA from the seabed or sea surface, the noise covariance matrix (required for beamforming) for an $N$-element array exhibits a Toeplitz-like structure ${ }^{3}$ with its first row given by the vector $\mathbf{s}_{N}=\left[\begin{array}{ll}s_{11} & s_{12} \cdots s_{1 N}\end{array}\right]$, where $s_{a b}$ is the coherence of the ambient-noise field between the $a$ th and $b$ th array sensors (the frequency dependency of $s_{a b}$ is omitted for brevity). The noise coherence can be written as

$$
s_{a b}=\int_{0}^{\pi / 2} \tilde{G}(\theta)\left[e^{i 2 \pi f \Delta(a-b) \sin \theta}+\tilde{R}\left(\theta_{b}\right) e^{-i 2 \pi f \Delta(a-b) \sin \theta}\right] \cos \theta d \theta,
$$

where $f$ is the frequency, $\Delta$ is the inter-element spacing, $\theta$ is the grazing angle measured at the VLA, $\tilde{R}\left(\theta_{b}\right)$ is the seabed power reflection coefficient, $\theta_{b}=\sin ^{-1}\left(\sin \theta c_{b} / c_{r}\right)$ is the grazing angle at the seabed, $c_{r}$ and $c_{b}$ indicate the water sound speed at the VLA and the water-sediment interface, respectively, and

$$
\tilde{G}(\theta) \frac{\sin \theta_{s}}{1-\tilde{R}\left(\theta_{b}\right) \tilde{R}_{s}\left(\theta_{s}\right)} .
$$

In Eq. (2), $\tilde{R}_{s}\left(\theta_{s}\right)$ is the power reflection coefficient at the air-water interface, $\theta_{s}=\sin ^{-1}$ $\left(\sin \theta \quad c_{s} / c_{r}\right)$ is the grazing angle at the sea surface, and $c_{s}$ is the water sound speed at the surface. With the change of variables $k_{z}=\left(f / c_{r}\right) \sin \theta$ (where $k_{z}$ is the vertical wavenumber), it can be shown 9 that $s_{a b}$ is the inverse Fourier transform of a band-limited signal with spectrum split into $\tilde{G}\left(k_{z}\right)$ and $\tilde{G}\left(-k_{z}\right) \tilde{R}\left(-k_{z}\right)$ over positive $\left(0<k_{z}<W_{f}\right)$ and negative $\left(-W_{f}<k_{z}<0\right)$ spatial frequencies (wavenumbers), where $W_{f}=f / c_{r}$.

Extension of $\mathbf{s}_{N}$ to represent a longer, uniformly spaced $N_{e}$-element array $\left(N_{e}>N\right)$ gives the coherence

$$
\mathbf{s}_{N_{e}}^{N}=\left(s_{11} s_{12} \cdots s_{1 N} s_{1(N+1)}^{N} \cdots s_{1 N_{e}}^{N}\right)
$$

where the upper index ${ }^{N}$ emphasizes that the elements $s_{1 b}^{N}$ (where $b>N$ ) are not observed (sampled) but rather are obtained from the $s_{1 b}(b \leq N)$ according to a specific algorithm. In contrast, $\mathbf{s}_{N_{e}}$ indicates a coherence measured with an actual $N_{e}$-element array. While the zero-padding $\operatorname{method}^{9}$ implemented by forcing $s_{1 b}^{N}=0$ for $N+1<b<N_{e}$ might be a reasonable approximation for fast-decaying coherences, a formal and more general extrapolation method for ambient noise coherence is described in the next section.

\section{Extrapolation of band-limited signals from partial observations}

The extrapolation of a band-limited signal $s(z)$ for $|z|>Z$ based on observations within the interval $|z|<Z$ can be achieved by projecting $s(z)$ onto the prolate spheroid functions (PSFs). ${ }^{10}$ In this paper, the array top element corresponds to $z=0$. The PSFs $\psi_{p}(z)(p=0, \ldots, \infty)$ form a band-limited complete orthogonal basis with spectra entirely contained in $-W_{f}<k_{z}<W_{f}$. The PSFs are obtained as the eigenfunctions of the integral equation

$$
\int_{-Z / 2}^{Z / 2} \frac{\sin \left((z-s) W_{f}\right)}{\pi(z-s)} \psi_{p}(s) d s=\mu_{p} \psi_{p}(z) ; p=0,1,2, \ldots
$$


where the eigenvalues $\mu_{p}$ can be thought as energy ratios that indicate the percentage of energy of the wavelet $\psi_{p}(z)$ localized at $|z|<Z / 2$ relative to its total energy. The PSFs are bi-orthogonal ${ }^{10}$ such that

$$
\int_{-Z / 2}^{Z / 2} \psi_{p}(z) \psi_{q}(z) d z=\left\{\begin{array}{ll}
0 & \text { if } p \neq q \\
\mu_{p} & \text { if } p=q ;
\end{array} \int_{-\infty}^{\infty} \psi_{p}(z) \psi_{q}(z) d z= \begin{cases}0 & \text { if } p \neq q \\
1 & \text { if } p=q .\end{cases}\right.
$$

In addition, $\psi_{p}(z)$ are band-limited for all $p$, and their Fourier transform has the property $^{11}$

$$
\int_{-\infty}^{\infty} \psi_{p}(z) e^{i 2 \pi f z} d z= \begin{cases}\nu_{p} \psi_{p}\left(\frac{Z}{2} \frac{f}{W_{f}}\right) & \text { if }|f| \leq W_{f} \\ 0 & \text { if }|f|>W_{f}\end{cases}
$$

where $\nu_{p}$ is a complex constant that can be found by enforcing Eq. (5). Equation (6) implies that by knowing $\psi_{p}(z)$ within the aperture, its Fourier transform can be constructed. Then taking the inverse Fourier transform gives $\psi_{p}(z)$ for $z$ outside the aperture.

Equation (5) allows expanding a function in terms of PSFs as

$$
s(z) \approx \sum_{p=0}^{P} a_{p} \psi_{p}(z) ; \quad a_{p}=\frac{1}{\mu_{p}} \int_{-Z / 2}^{Z / 2} s(z) \psi_{p}(z) d z,
$$

which, in theory, accomplishes perfect extrapolation of $s(z)$ beyond $|z|<Z / 2$ as $P \rightarrow \infty$. In practice, this extrapolation can be affected by numerical artifacts for small $\mu_{p}$, so a threshold for the number of terms $P$ in Eq. (7) must be set. In this paper, computation of the PSFs is performed by discretizing Eq. (4); this yields the discrete prolate spheroidal sequences ${ }^{11}$ for $|z|<Z / 2$. The discrete equation can be solved by commercial software packages such as the MATLAB script "dpss.m." The property in Eq. (6) is then used with the inverse FFT to obtain the PSFs for $|z|>Z / 2$.

\section{Ambient-noise coherence extrapolation}

This section demonstrates the extrapolation approach in Eq. (7) applied to simulated and experimental noise coherence functions.

\subsection{Simulated data}

Table 1 shows the parameters of two environments with a seabed consisting of three and five sediment layers (including a bottom half-space). For both environments, the water depth is $130 \mathrm{~m}$. These parameters are input to Eq. (1) to obtain the coherence function $s(z)$ at $f=750 \mathrm{~Hz}$ with constant water sound speed $c_{r}=1500 \mathrm{~m} / \mathrm{s}$.

Table 1. Parameters for three- and five-layer models used to generate simulated ambient-noise data.

\begin{tabular}{lllllllll}
\hline \hline \multirow{2}{*}{ Layer } & \multicolumn{7}{c}{$c_{l}(\mathrm{~m} / \mathrm{s})$} & \multicolumn{2}{c}{$\rho_{l}\left(\mathrm{~kg} / \mathrm{m}^{3}\right)$} & \multicolumn{2}{c}{$\alpha_{l}(\mathrm{~dB} / \lambda)$} & \multicolumn{2}{c}{$h_{l}(\mathrm{~m})$} \\
\hline 1 & 1520 & 1480 & 1542 & 1300 & 0.2 & 0.25 & 0.8 & 0.44 \\
2 & 1750 & 1530 & 2306 & 1760 & 1.5 & 0.2 & 0.8 & 0.51 \\
3 & 1598 & 1621 & 1813 & 1400 & 0.4 & 0.2 & $\infty$ & 0.18 \\
4 & - & 1552 & - & 1500 & - & 0.2 & - & 1.75 \\
5 & - & 1682 & - & 1960 & - & 0.1 & - & $\infty$ \\
\hline \hline
\end{tabular}



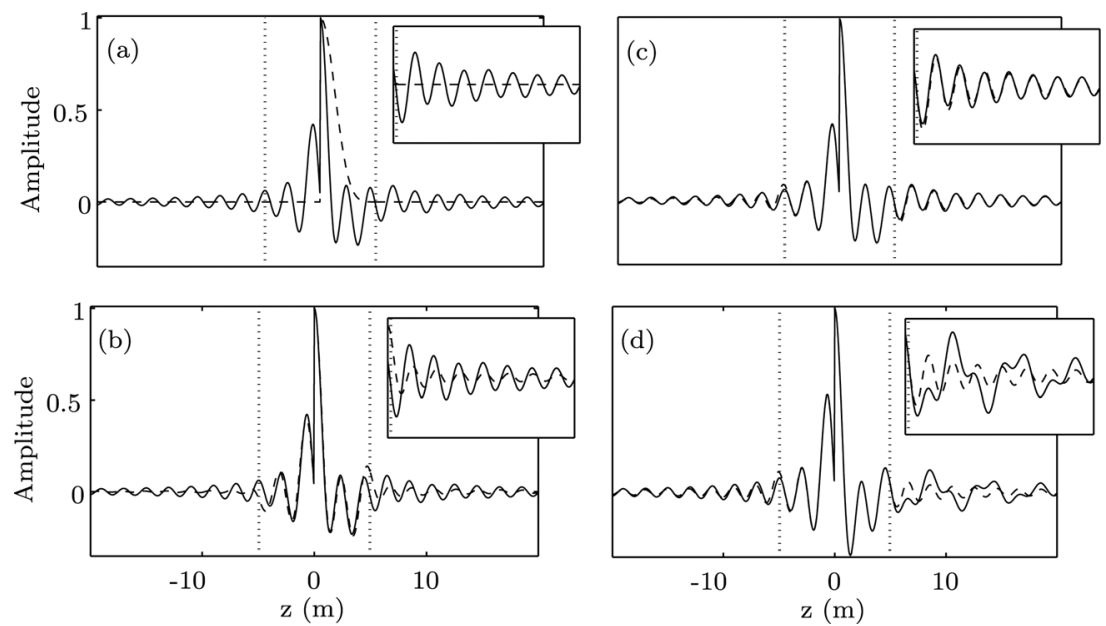

Fig. 1. Extrapolation results (dashed lines) for ambient-noise coherence at $f=750 \mathrm{~Hz}$ (solid lines) according to Eq. (7). Panels (a), (b), and (c) show the extrapolation results for $P=0,9$, and 11 , respectively, for the coherence corresponding to a three-layer sediment profile. Panel (d) shows the results corresponding to a five-layer sediment profile for $P=11$. The input to the extrapolation is the segment of coherence within the observation aperture (indicated as vertical dotted lines). Inset plots provide zoomed-in views of the extrapolated results (dotted lines included as reference). The results corresponding to the real (imaginary) part are shown for $z>0(z<0)$.

Figures 1(a)-1(c) show the extrapolation of $s(z)$ for the three-layer environment for increasing $P$. The simulated coherence (shown as solid lines) represents what would be "measured" with a 100-element array with inter-element spacing of $5 \mathrm{~cm}(4.95 \mathrm{~m}$ aperture). The real (and even) part of the coherence is shown for $z>0$, while the imaginary (and odd) part is shown for $z<0$. Notice that the coherence functions are normalized to unit maximum for display purpose. Because the extrapolated results are better for the imaginary part than for the real part of the coherence, zoomed-in views of the extrapolation are provided only for the latter. This convention will be followed throughout this paper. The extrapolation results are shown as dashed lines: Figs. 1(a)-1(c) correspond to $P=0,9$, and 11 in Eq. (7). For $P=0$, the real part is coarsely approximated by $\psi_{0}(z)$, and the representation of the true covariance is inaccurate even within the aperture. For $P=9$ (which includes the PSFs with $\mu_{p}>0.5$ ), the extrapolated coherence matches well within the aperture, and it predicts the oscillatory behavior of the covariance for $|z|>Z / 2$. Adding two more PSFs (which corresponds to including all PSFs with $\mu_{p}>0.06$ ) to the summation gives even more accurate results shown in Fig. 1(c). In this paper, selection of the number of terms $P$ was done based on minimizing the difference between the extrapolated coherence and the coherence measured at the aperture. Similar results can be obtained by plotting $\sqrt{\sum_{p=1}^{P}\left|a_{p}\right|^{2}}$ in a $\log \log$ scale against the L2 norm of the error within the aperture given by $\sqrt{\sum_{t=1}^{N}\left|s_{1 t}^{N}(P)-s_{1 t}\right|^{2}}$ [notice that $s_{1 t}^{N}(P)$ emphasizes the dependence of the extrapolated coherence on $P$ ]. This plot results in an L-shaped trace that provides a graphical tool to select the value $P_{\text {elbow }}$ corresponding to the L-shape elbow. The rationale of the approach inspired in the L-curve method is that even though the coefficients $\left|a_{p}\right|$ tend to grow (due to normalization by decreasing eigenvalues $\mu_{p}$ ), their contribution to minimizing the errors $\left|s_{1 t}^{N}(P)-s_{1 t}\right|$ is not significant for $P>P_{\text {elbow }}$. In addition, as pointed out previously, it is a good practice to limit $P$ because problems of numerical instability can occur by adding more terms to Eq. (7) due to inaccuracies in determining the projection coefficients $a_{p}$.

Figure 1(d) shows extrapolation results for a more challenging signal $s(z)$, obtained from the coherence function at $f=750 \mathrm{~Hz}$ of the five-layer sediment profile 
described in Table 1. In this case, the real part of the coherence exhibits more structure in its tail, as opposed to the decaying-envelope behavior of $s(z)$ in Figs. 1(a)-1(c). Even though the extrapolation results are less accurate, the trend of oscillations is estimated correctly. Similar extrapolation results were obtained for simulated data corresponding to more sparse VLAs with $\Delta=0.5 \mathrm{~m}$ inter-element spacing.

Throughout this paper, the reason for obtaining better extrapolation results for the imaginary part of the coherence can be seen from Eqs. (1) and (2): Consider that the Fourier transform of the coherence, $\mathscr{F}\left(\mathbf{s}_{\mathrm{ab}}(z)\right)$, is real ${ }^{9}$ and can be expressed as a summation of even and odd functions. Because $\tilde{G}\left(k_{z}\right)=\tilde{G}\left(-k_{z}\right)$ and $\tilde{R}\left(k_{z}\right)=\tilde{R}\left(-k_{z}\right)$, the even function [responsible for the real part of $s_{a b}(z)$ ] is strongly dependent on the seabed reflection coefficient, which introduces complicated features, while the effect of the seabed in the odd function [which gives the imaginary part of $\left.s_{a b}(z)\right]$ tends to cancel out due to symmetry, giving a smoother (easier to extrapolate) function.

\subsection{Experimental data}

The coherence extrapolation was applied to measured data from the BOUNDARY2003 experiment, ${ }^{12}$ collected by the NATO-STO Centre for Maritime Research and Experimentation (CMRE formerly NATO Undersea Research Centre). The aperture used in the experiment consists of a 32-element drifting array of omnidirectional pressure sensors with $\Delta=0.18 \mathrm{~m}$ inter-element spacing. The data were collected in a region with water depth of approximately $131 \mathrm{~m}$ at a sampling rate of $12 \mathrm{kHz}$, and the frequency-dependent data covariance was computed from snapshots recorded over a 5-min interval. The $32 \times 32$ element covariance matrix was made Toeplitz by taking the mean along diagonals, and this is taken as a reference (i.e., ground truth) in this section on the understanding that it differs from the true covariance, which could only be measured accurately with a larger (and denser) aperture array.

Figure 2 shows the extrapolation for $N=12$ at frequencies $f=703,797,891$, 1008, 1102, and $1195 \mathrm{~Hz}$. The extrapolated covariance with $N_{e}=32$ is in agreement with the $N_{e}$-element reference covariance, and they exhibit similar behavior as the simulated results obtained in Sec. 4.1. Similar to Figs. 1(c) and 1(d), the extrapolated imaginary part $(z<0)$ closely matches the reference, and the extrapolated real part $(z>0)$ follows the oscillatory pattern of the reference but is less accurate. In addition, the reconstructed coherence within the observation aperture $(|z|<Z / 2)$ closely matches the reference.

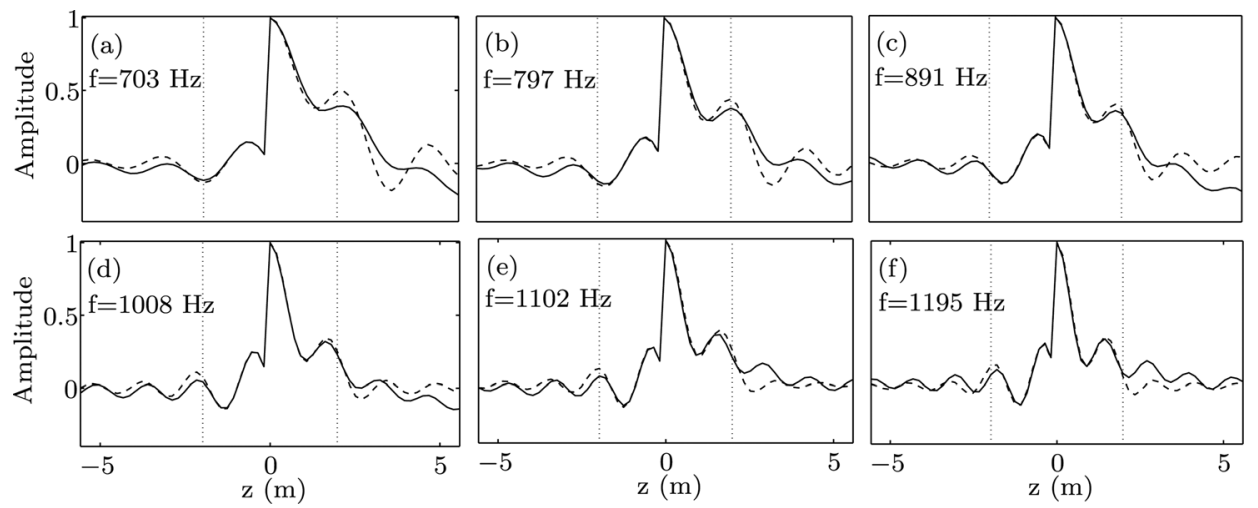

Fig. 2. Coherence extrapolation for measured data from the BOUNDARY2003 experiment (Ref. 12) at six frequencies from $f=703$ to $1195 \mathrm{~Hz}$. Solid and dashed lines represent $\mathbf{s}_{N_{e}}$ (reference) and $\mathbf{s}_{N_{e}}^{N}$ (extrapolated) coherences, respectively, where $N=12$ and $N_{e}=32$. Vertical dotted lines represent the limits of the observation aperture. 


\section{Summary}

This paper developed a procedure for the extrapolation of spatially band-limited underwater ambient-noise measurements based on projections onto a prolate spheroid function basis. The algorithm offers a formal approach to synthetic array processing of surface-generated ambient noise as an alternative to more heuristic methods such as coherence zero-padding. ${ }^{9}$ The true coherence within the aperture can be accurately represented by $P$ orthonormal prolate spheroid functions as shown here for experimental and simulated data. Outside the aperture, the algorithm successfully estimates the general oscillation pattern of the noise coherence.

A potential application of the extrapolated results that is subject of further research is on improving the resolution of seabed parameters estimated by geoacoustic inversion. ${ }^{4,8}$ This is plausible as long as the behavior of the extrapolated coherence (or quantities derived from this coherence such as the seabed reflection coefficient input to inversion algorithms $\mathrm{s}^{8}$ ) can be predicted and accounted for in the forward model used in the inversion method. This can be accomplished by applying the PSF basis not only to the measured data (i.e., for extrapolation) but also to the coherence model ${ }^{8}$ used to generate data replicas while searching the parameter space during execution of the inversion algorithm.

\section{Acknowledgments}

The authors gratefully acknowledge the support of the Office of Naval Research postdoctoral fellowship and the Ocean Acoustics Program (ONR-OA Code 3211) as well as the Natural Sciences and Engineering Research Council (NSERC) of Canada. We would also like to thank the NATO-STO Centre for Maritime Research and Experimentation (CMRE formerly NATO Undersea Research Centre), Chris Harrison, and Peter Nielsen, for providing the BOUNDARY2003 data.

\section{References and links}

${ }^{1}$ W. A. Kuperman and F. Ingenito, "Spatial correlation of surface generated noise in a stratified ocean," J. Acoust. Soc. Am. 67, 1988-1996 (1980).

${ }^{2}$ F. Ingenito and S. N. Wolf, "Site dependence of wind-dominated ambient noise in shallow water," J. Acoust. Soc. Am. 85, 141-145 (1989).

${ }^{3}$ C. H. Harrison, "Formulas for ambient noise level and coherence," J. Acoust. Soc. Am. 99, 2055-2066 (1996)

${ }^{4}$ C. H. Harrison and D. G. Simons, "Geoacoustic inversion of ambient noise: A simple method," J. Acoust. Soc. Am. 112, 1377-1389 (2002).

${ }^{5}$ M. Siderius, C. H. Harrison, and M. B. Porter, "A passive fathometer technique for imaging seabed layering using ambient noise,” J. Acoust. Soc. Am. 120, 1315-1323 (2006).

${ }^{6} \mathrm{M}$. Siderius and C. H. Harrison, "High-frequency geoacoustic inversion of ambient noise data using short arrays," AIP Conf. Proc. 728, 22-31 (2004).

${ }^{7}$ J. E. Quijano, S. E. Dosso, J. Dettmer, L. M. Zurk, M. Siderius, and C. H. Harrison, "Bayesian geoacoustic inversion using wind-driven ambient noise,” J. Acoust. Soc. Am. 131, 2658-2667 (2012).

${ }^{8}$ J. E. Quijano, S. E. Dosso, J. Dettmer, L. M. Zurk, and M. Siderius, "Transdimensional geoacoustic inversion of wind-driven ambient noise," J. Acoust. Soc. Am. 133, EL47-EL53 (2013).

${ }^{9}$ M. Siderius, L. Muzi, C. H. Harrison, and P. L. Nielsen, "Synthetic array processing of ocean ambient noise for higher resolution seabed bottom loss estimation," J. Acoust. Soc. Am. 133, EL149-EL155 (2013).

${ }^{10}$ D. Slepian and H. O. Pollak, "Prolate spheroidal wave functions, Fourier analysis and uncertainty," Bell System Tech. J. 40, 43-64 (1961).

${ }^{11}$ D. Slepian, "Some comments on Fourier analysis, uncertainty, and modeling," SIAM Rev. 25, 379-393 (1983).

${ }^{12}$ C. H. Harrison, "Performance and limitations of spectral factorization for ambient noise sub-bottom profiling,” J. Acoust. Soc. Am. 118, 2913-2923 (2005). 